\title{
OS BENEFÍCIOS DO EXERCÍCIO FÍSICO EM PACIENTES COM DOENÇA RENAL CRÔNICA: UMA REVISÃO BIBLIOGRÁFICA
}

\author{
France Willian Ávila do Nascimento ${ }^{1}$ \\ Andréa Araújo dos Santos ${ }^{2}$
}

RESUMO: A incidência da doença renal crônica vem aumentando a cada ano, é necessária uma atenção especial por parte do governo para que essa doença seja prevenida e tratada de maneira adequada por quem já estar com a doença. Os indivíduos com insuficiência renal crônica são considerados um grupo especial que necessita de um acompanhamento diferenciado quando se submetem a realização de exercícios físicos. A doença renal crônica não provoca apenas problemas físicos mais também problemas psicológicos relacionado a medicação e a rotina de tratamento que não é muito estimulante. O objetivo deste estudo é salientar a importância do exercício Físico para esta população. De acordo com os estudos abordados neste trabalho, o exercício demostrou ser um aliado tanto na melhora da mobilidade e do condicionamento físico quanto na melhora da autoestima dos pacientes submetidos ao tratamento de hemodiálise.

Palavras-chave: Hemodiálise. Doença renal. Exercício físico.

ABSTRACT: The incidence of chronic kidney disease is increasing every year, special attention is needed by the government so that this disease is prevented and treated properly by those who already have the disease. Individuals with chronic renal failure are considered a special group that needs differentiated monitoring when undergoing physical exercise. Chronic kidney disease not only causes physical problems but also psychological problems related to medication and treatment routine that is not very stimulating. The objective of this study is to emphasize the importance of physical exercise for this population. According to the studies addressed in this work, exercise proved to be an ally both in improving mobility and physical conditioning and in improving the self-esteem of patients undergoing hemodialysis treatment.

Keywords: Hemodialysis. Kidney disease. Physical exercise.

\footnotetext{
${ }^{\text {I }}$ Pós- graduado em Fisiologia do Exercício, possui graduação em Educação Física-Licenciatura pela Universidade Federal do Acre (2019). Tem experiência na área de Educação Física, com ênfase em Musculação (2016). E-mail: franceacre@hotmail.com.

${ }^{2}$ Formação acadêmica: Bacharelado em enfermagem pela Universidade Federal do Acre.
} 


\section{INTRODUÇÃO}

De acordo com da Sociedade Brasileira de Nefrologia, a estimativa da doença renal crônica no mundo é de $7,2 \%$ para as pessoas a cima de 30 anos e $28 \%$ a $46 \%$ em indivíduos acima de 64 anos. No Brasil, a estimativa é de que mais de dez milhões de pessoas tenham a doença. (KUPSKE, 2020).

Fisiologicamente o corpo humano, para manter o seu equilíbrio da homeostase, necessita que haja um ótimo funcionamento de todos os seus órgãos, vitais e principalmente dos rins, pois são eles os responsáveis pela eliminação de catabólicos do corpo através do controlo de líquidos e também pela filtração do sangue, mantendo a função vital do organismo (SANTOS et al, 2017).

De acordo com o Ministério da Saúde o rim possui várias funções vitais e que todas elas são fundamentais para o organismo se manter vivo e funcionando. As principais delas são:

- $\quad$ Excreção de produtos finais de diversos metabolismos;

- Controle do metabolismo acidobásico;

- Controle do equilíbrio hidroeletrolítico;

- Controle da pressão arterial.

- $\quad$ Produção de hormônios;

A evolução da doença é bastante lenta e em alguns casos silenciosa, o que possibilita o organismo a passar por diferentes alterações que vão surgindo de acordo com o avanço da doença, e por isso existe caso em que os sintomas surjam apenas quando a doença se encontra no último estágio, chamado pela literatura como fase pré-dialítica (SANTOS, 2017).

Os indícios da doença renal crônica é possível identificar, através de análises clínicas, onde é analisado os níveis de fósforo e de potássio, e fatores que dão origem a anemia e acidose. Com tudo, existem outros sintomas, como alguns sinais desnutrição, emagrecimento, hipertensão, os ossos ficam mais frágeis e dentre outros fatores. Todos esses fatores contribuem para o surgimento de outras alterações como a diminuição da massa muscular, no entanto, devido à retenção de líquidos provocada pelo mau funcionamento dos rins, a perda de peso pode ser um sintoma 
que não é completamente evidente visto que o peso poderá manter-se mais na maioria dos casos o peso aumenta (SANTOS, 2017).

Segundo Silva (2019), quando a função renal se encontra inferior a 50\% pode surgir alguns sintomas mais visíveis como uma menor produção de urina, mas a elevada necessidade de urinar ainda é mantida, as mãos e pernas ficam inchadas, falta de ar, o paciente passa a ter dificuldade em dormir, perda de apetite, náuseas e vómitos, hipertensão e sensação de frio e fadiga.

A doença renal crônica é dividida em cinco estágios de acordo com a gravidade de evolução relacionado ao grau de filtração glomerular. Quando é alcançado o estágio mais avançado é necessário que o paciente inicie um tratamento de hemodiálise que tema função de substituição renal, (ANDRADE, et al, 2018).

O tratamento por hemodiálise faz com que a rotina destes doentes seja mais restrita devido as limitações a que estes indivíduos estão submetidos após o início do tratamento. O tempo de duração de uma sessão de hemodiálise é bastante demorado e isso faz com que as atividades diárias com as atividades corporais, domésticas e recreativas sejam comprometidas, o que leva estes doentes a adotar comportamentos menos saudáveis levando ao sedentarismo (MARCHESAN et al, 2017).

Desta forma, O Profissional de Educação Física surge com um papel fundamental na prestação de serviços para esta população e precisam estar preparados para se adaptar as limitações progressivas desta doença que podem ser encaradas como justificativa para a inatividade física, a prática regular de exercícios físicos é aspecto fundamental para a promoção da saúde destas pessoas.

\section{FUNDAMENTAÇÃO TEÓRICA}

A doença renal é caracterizada pela perda gradual ou imediata de uma das funções do órgão vital que é o rim, ou seja, é a perca da função do rim. A evolução da doença renal crônica é assintomática, ou seja, é difícil de se detectar, desta forma o paciente acaba fazendo com que o diagnóstico seja feito durante um estado mais avançado da doença diminuindo de forma significativa as chances de cura definitiva (MARINHO, 2020). 
De acordo com a American Diabetes Association (2013), a partir do momento que as artérias existentes nos rins são sujeitas a elevados valores da pressão arterial, com o passar dos anos acabam perdendo a capacidade de filtrar o sangue, pois a principal função do rim é remover alguns catabólicos e o excesso de água presente no organismo (MEDEIROS, 2016).

Assim, a disfunção renal leva a uma redução dessa capacidade que são fundamentais para o organismo se manter vivo e funcionando. Nesse caso, o principal tratamento imediato é o procedimento de hemodiálise (MARINHO, 2020).

Hemodiálise é um procedimento médico que se utiliza de uma máquina que tem uma função de limpa e filtra o sangue, ou seja, a máquina de diálise faz a função renal do paciente. Este procedimento faz uma filtragem do sangue eliminando tanto resíduos prejudiciais à saúde quanto vitaminas e minerais que são benéficos para o funcionamento perfeito do corpo humano, tentando manter o equilíbrio de substâncias como sódio, potássio, uréia e creatinina. As sessões de hemodiálise são realizadas geralmente em clínicas especializadas ou hospitais (MARINHO, 2020).

De acordo com a Sociedade brasileira de nefrologia a hemodiálise é indicada para pacientes que possuem insuficiência renal aguda ou crônica em estado mais grave. A indicação para iniciar esse tratamento é feita por recomendação médica especializada em doenças renais (o nefrologista), que fará a seguinte avaliação:

- Consulta médica, investigando os seus sintomas e examinando o seu corpo;

- Dosagem de ureia e creatinina no sangue;

- $\quad$ Dosagem de potássio no sangue;

- Dosagem de ácidos no sangue;

- Quantidade de urina produzida durante um dia e uma noite (urina de 24 horas);

- Cálculo da porcentagem de funcionamento dos rins (clearance de creatinina e uréia);

- Avaliação de anemia (hemograma, dosagem de ferro, saturação de ferro e ferritina);

- Presença de doença óssea. 
$\mathrm{Na}$ hemodiálise a máquina recebe o sangue do paciente diretamente da artéria braquial, que pode ser um feito através de um cateter (tubo) ou uma fístula arteriovenosa, e depois é impulsionado por uma bomba até o filtro de diálise (dialisador) (BERNARDO, 2019).

No dialisador o sangue é exposto as soluções que retira as substancias que estão acumuladas na corrente sanguínea deixando sangue limpo para o paciente pelo acesso vascular. O tempo de duração de uma sessão de hemodiálise varia de acordo com o estado clínico do paciente, geralmente a sessão tem duração de quatro horas, com frequência de três ou quatro vezes por semana (BERNARDO, 2019).

Há ocasiões clínicas que o paciente realiza o tratamento com duração de 3 a 5 horas por sessão e pode ser feita 2, 3, 4 vezes por semana ou até mesmo diariamente nos casos mais graves. O paciente em tratamento não deve faltar as suas sessões pois o seu estado clinico pode se agravar levando em alguns casos até o óbito (PRETTO, 2020).

A qualidade de vida desta população sofre muitas alterações negativas como por exemplo a desnutrição devido as restrições a alguns alimentos, alterações cognitivas relacionadas a rotinas e dependência da máquina de hemodiálise, alterações na qualidade do sono, e em alguns casos há pacientes que apresentam elevados níveis de depressão.

Afsar, et al. (2018), em seu estudo verificou que a qualidade de vida de pacientes renais sofre muitas alterações com alterações cognitivas, desnutrição, alterações no sono pois tem paciente que moram distante da clínica e tem que acordar muito cedo para não perder o horário do tratamento, além de que apresentam níveis de depressão.

A doença renal crônica afeta diretamente a qualidade de vida do paciente, por vezes de maneira mais intensa que outras doenças crônicas. A diminuição da capacidade funcional, tem como consequência a baixa tolerância ao exercício físico e por isso as atividades físicas da vida diária são outras alterações que surgem no decorrer do tratamento da hemodiálise (REBOREDO et al, 2007).

O exercício físico é toda atividade física sistematizada ou padronizada com tempo de duração pré-estabelecidos. Por isso, conta com uma duração de tempo de 
duração específico, com sequências de movimentos para diferentes partes do corpo e proporciona o aumento das aptidões físicas, como flexibilidade, força, resistência e dentre outras. O exercício físico é um aliado importante de quem quer aumentar a saúde do sistema cardiovascular além de estimular o sistema imunológico a longo prazo, que passa a ter mais força para combater doenças (COELHO, 2008).

O exercício físico em pacientes submetidos à hemodiálise é muito importante, para esses pacientes pois os mesmos apresentam acentuada redução da funcionalidade e do condicionamento, o que interfere de forma negativa na qualidade de vida dessas pessoas. Contudo, os benefícios, o tipo de exercício mais adequado e parâmetros como intensidade, frequência e duração não estão bem esclarecidos para essa população (COELHO, 2008).

Segundo Nery et al, (2009), este tipo de população apresenta baixo condicionamento físico que é verificada pelas alterações na coordenação muscular, diminuição da força e na composição corporal, que na maioria estão acima do peso recomendado.

O exercício físico funciona como uma ferramenta importante no combate ao sedentarismo, no qual é um estilo de vida que provoca outros tipos de patologias além à doença renal crónica. Assim, manutenção da aptidão física dos pacientes em hemodiálise é essencial para evitar que ocorra a piora da sua qualidade de vida, uma vez que a aptidão física é fator que está diretamente relacionado com a mortalidade e com a qualidade de vida de uma pessoa (KUTSUNA, et al, 2010).

Estudos como o de Cantareli (2009), que aplicaram 5 meses de treinamento de força e resistência nos membros inferiores durante a realização da hemodiálise, demonstrou um aumento da força muscular para os extensores do joelho (JURIATTI, 2020).

\section{MATERIAL E MÉTODOS}

Tratar-se de uma revisão bibliográfica realizada através da identificação, seleção e leitura de estudos referentes ao tema embasada em artigos que foram de fundamental importância para a compreensão e construção do presente trabalho, esta estratégia possibilita analisar, rever interpretar e, até mesmo, criticar considerações 
teóricas, possibilitando criar novas propostas de explicação e de compreensão dos fenômenos e fatos das mais diferentes áreas do conhecimento cientifico (LAKATOS, 1991).

Assim como também, foram abordadas as recomendações da Sociedade brasileira de nefrologia que faz diversos levantamento sobre pacientes renais no Brasil e que busca criar protocolos para proporcionas um tratamento adequado para os pacientes com doença renal crônica.

\section{RESULTADOS E DISCUSSÃO}

De acordos com os artigos pesquisados o exercício físico praticado por pacientes com doença renal crônica submetidos ao tratamento de hemodiálise mostrou-se ser benéfico para essa população. Segundo o estudo realizado por Rosa, et al. (2018), onde realizou 12 semanas de exercícios de resistência progressiva e 12 semanas de exercícios controlados em pacientes que realizava a hemodiálise, verificou-se que a massa magra total aumentou diminuindo o percentual de gordura no corpo.

Já no estudo realizado por Reboredo, et al (2007), após realizar por doze semanas treino de força em pacientes renais crónicos submetidos a hemodiálise, constatou-se o aumento da força muscular do quadríceps. Ainda neste estudo, os autores citam outro estudo que foi avaliado o efeito do exercício aeróbico que foi realizado durante ro semanas de treino aeróbio e após o período de treino, a força de membros inferiores aumentou cerca de $16 \%$ e a resistência muscular de membros inferiores aumentou $53 \%$.

Outro estudo que abordou o treino de força e resistência aeróbica foi o estudo realizado por Nascimento, et al (2012), que em cinco meses de treino de força e resistência muscular, mostrou aumento da força nos extensores do joelho, assim como o treino aeróbio também provoca melhorias significativas na força muscular neste tipo de população.

$\mathrm{Na}$ revisão de literatura realizada por Dias (2019), dos 22 estudos analisados concluiu-se entre eles o aumento da resistência aeróbica, melhora na qualidade de vida, melhora na qualidade do sono, aumento da capacidade funcional, possibilitando 
aos pacientes mais independência e autonomia na realização de tarefas diárias, além da melhora nos testes aplicados pelos pesquisadores como caminhada, teste de sentar e levantar, além notar-se a diminuição da dor após as sessões de Hemodiálise.

Pode-se notar que a maiorias dos estudos estão relacionados na melhora do condicionamento relacionado a resistência aeróbica e ganhos de forçar, essas qualidades físicas tem papel fundamental para a realização de atividades diárias de qualquer ser humano.

Já no estudo realizado por De Lima (2019), que realizou um programa de exercícios, desenvolvido três vezes por semana, durante 40 minutos em hemodiálise, por oito semanas, ao final do estudo concluiu-se que o exercício melhorou a resistência as dores musculares após as sessões de Hemodiálise.

$\mathrm{Na}$ pesquisa realizada por Willian (2019) concluiu que o exercício proporcionou uma redução das dores musculares que são características desta população devido a inércia do tratamento que em média tem 4 horas de duração sendo realizado pelo menos 3 vezes na semana. $O$ paciente faz o tratamento em decúbito dorsal, consequentemente com anos de tratamentos alguns desvios posturais vão ocorrer causando dores.

\section{CONCLUSÃO}

Diante das pesquisas abordas conclui-se que o exercício físico supervisionado por um profissional de Educação Física sempre respeitando a individualidade biológica de cada paciente mostrou-se benéfico para esta população.

Os resultados demonstram a eficaz do exercício no ganho de força, resistência, flexibilidades e dentre outras qualidades físicas que facilitaram no desempenho de atividades diárias, além de melhorar o humor promovendo uma melhor qualidade de vida desses pacientes. Dentre os exercícios mais eficazes para esta população encontrou-se o exercício aeróbico realizado por um cicloergômetro acoplado na maca durante a realização do tratamento de hemodiálise.

É muito importante a realização de novos estudos que abordem a eficaz do exercício tanto no seu aspecto físico quanto psicológico, comparando entre um grupo 
que participa do programa de exercícios com os que não fazem nenhuma atividade física.

\section{REFERÊNCIAS}

ANDRADE, Joyce C. et al. Doença Renal Crônica Afeta Negativamente a Composição Corporal, Qualidade de Vida, Perfil Lipídico e a Aptidão Física de Pacientes em Tratamento de Hemodiálise. Motricidade, v. 14, 2018.

AFSAR, Baris et al. The impact of exercise on physical function, cardiovascular outcomes and quality of life in chronic kidney disease patients: a systematic review. International Urology and Nephrology, v. 50, n. 5, p. 885-904, 2018.

COELHO, Douglas Martins; RIBEIRO, José Márcio; SOARES, Danusa Dias. Exercícios físicos durante a hemodiálise: uma revisão sistemática. J Bras Nefrol, v. 30, n. 2, p. 88-98, 2008.

DE LIMA, Fabiano F. et al. Avaliação funcional pré e pós-programa de exercício físico de pacientes em hemodiálise. Medicina (Ribeirão Preto. Online), v. 46, n. I, p. 24-35, 2013.

DIAS, Henrique Souza. O efeito do exercício físico em pacientes submetidos ao tratamento de hemodiálise. Revista Brasileira de Fisiologia do Exercício, v. I8, n. I, p. 58-63, 2019.

JURIATTI, Anelize. Efeitos de um protocolo de fisioterapia respiratória e motora em pacientes renais crônicos durante a hemodiálise: ensaio clínico randomizado e controlado. Programa de Pós-Graduação em Ciência da Saúde, 2020.

KUTSUNA, Toshiki et al. Physical activity is necessary to prevent deterioration of the walking ability of patients undergoing maintenance hemodialysis. Therapeutic Apheresis and dialysis, v. I4, n. 2, p. 193-200, 2010.

KUPSKE, Juliedy Waldow et al. INSUFIÊNCIA RENAL CRÔNICA E EXERCÍCIO FÍSICO. Plataforma de Submissão de Trabalhos e Anais de Eventos da Unicruz, v. I, n. 2, 2020.

LAKATOS, Eva Maria; MARCONI, M. de A. Metodologia científica. $2^{\underline{a}}$ edição. São Paulo: Atlas, I991.

MARINHO, Daliane Ferreira et al. Capacidade funcional e qualidade de vida na doença renal crônica. Revista Pesquisa em Fisioterapia, v. Io, n. 2, p. 212-219, 2020.

MARCHESAN, Moane et al. Percepção de pacientes em hemodiálise sobre os benefícios e as modificações no comportamento sedentário após a participação em um programa de exercícios físicos. Revista Brasileira de Ciências do Esporte, v. 39, n. 3, p. 314-321, 2017

MONTAÑA, Alexandra et al. Sistemas e-health para el tratamiento de la diabetes. Revista vínculos, v. II, n. 2, p. III-I26, 20I4.

MEDEIROS, Thaisy Garcia de Oliveira et al. Prevalência de pré-diabetes e fatores de risco para diabetes mellitus tipo 2 em adolescentes e características associadas a esta condição. 2016.

NERY, Rosane Maria; ZANINI, Maurice. Efeitos de um programa de 12 semanas de exercícios físicos sobre a capacidade funcional e a qualidade de vida de pacientes com doença renal crônica em hemodiálise. J. Bras. Nefrol., v. 31, n. 2, p. 151-153, 2009. 
NASCIMENTO, Leilane Cristielle de Alencar; COUTINHO, Érika Bona; SILVA, Kelson Nonato Gomes da. Efetividade do exercício físico na insuficiência renal crônica. Fisioterapia em Movimento, v. 25 , n. I, p. 231-239, 2012.

PRETTO, Carolina Renz et al. Depressão e pacientes renais crônicos em hemodiálise: fatores associados. Revista Brasileira de Enfermagem, v. 73, 2020.

REBOREDO, Maycon de Moura et al. Exercício físico em pacientes dialisados. Revista Brasileira de Medicina do Esporte, v. 13, n. 6, p. 427-430, 2007.

ROSA, Clara Suemi da Costa et al. Effect of continuous progressive resistance training during hemodialysis on body composition, physical function and quality of life in end-stage renal disease patients: a randomized controlled trial. Clinical rehabilitation, v. 32, n. 7, p. 899-908, 2018.

REBOREDO, Maycon de Moura et al. Exercício físico em pacientes dialisados. Revista Brasileira de Medicina do Esporte, v. 13, n. 6, p. 427-430, 2007.

SOCIEDADE BRASILEIRA DE NEFROLOGIA; SOCIEDADE BRASILEIRA DE NEFROLOGIA. Censo 20II. Recuperado em, v. I, 2014.

SANTOS, Bianca Pozza dos et al. Doença renal crônica: relação dos pacientes com a hemodiálise. ABCS health sci, p. 8-14, 2017..

SANTOS, Bianca Pozza dos et al. Doença renal crônica: relação dos pacientes com a hemodiálise. ABCS health sci, p. 8-14, 2017.

SILVA, Rute Isabel Gafaniz da. Efeito de um programa de exercício físico em pacientes em hemodialise. 2019. Dissertação de Mestrado. Universidade de Évora.

WILLIAN, France; GALISTEU, Rafaela Ester. Efeitos do exercício físico aeróbico na pressão arterial de pacientes com doença renal crônica em hemodiálise. South American Journal of Basic Education, Technical and Technological, v. 6, n. 2, p. 325-334, 2019. 